\title{
Empathy and its Modulation in a Virtual Human
}

\author{
Hana Boukricha ${ }^{1}$, Ipke Wachsmuth ${ }^{1}$, Maria Nella Carminati ${ }^{2}$, and Pia Knoeferle ${ }^{2}$ \\ 1 A.I. Group, Faculty of Technology, Bielefeld University, 33594 Bielefeld, Germany \\ \{hboukric,ipke\}@techfak.uni-bielefeld.de \\ 2 Cognitive Interaction Technology (CITEC), Bielefeld University, Morgenbreede 39, \\ Gebäudeteil H1, 33615 Bielefeld, Germany \\ mcarmina@techfak.uni-bielefeld.de, knoeferl@cit-ec.uni-bielefeld.de
}

\begin{abstract}
Endowing artificial agents with the ability to empathize is believed to enhance their social behavior and to make them more likable, trustworthy, and caring. Neuropsychological findings substantiate that empathy occurs to different degrees depending on several factors including, among others, a person's mood, personality, and social relationships with others. Although there is increasing interest in endowing artificial agents with affect, personality, and the ability to build social relationships, little attention has been devoted to the role of such factors in influencing their empathic behavior. In this paper, we present a computational model of empathy which allows a virtual human to exhibit different degrees of empathy. The presented model is based on psychological models of empathy and is applied and evaluated in the context of a conversational agent scenario.
\end{abstract}

\section{Introduction}

Research on empathic artificial agents corroborates the role of empathy in improving artificial agents' social behavior. For instance, it has been shown that empathic virtual humans can reduce stress levels during job interview tasks [17] and that empathic agents are perceived as more likable, trustworthy, and caring [7]. Furthermore, it has been found that empathic virtual humans can evoke empathy in children and can thus teach them to deal with bullying situations [16] and that a virtual human's empathic behavior also contributes to its ability to build and sustain long-term socio-emotional relationships with human partners [3]. However, it has been shown that in a competitive card game scenario, empathic emotions can increase arousal and induce stress in an interaction partner [1]. In line with neuropsychological findings [8] that humans empathize with each other to different degrees depending on their mood, personality, and social relationships with others, the modulation of a virtual human's empathic behavior through such factors would allow for a more adequate empathic behavior in the agent across different interaction scenarios. Although there is increasing interest in endowing artificial agents with affect, personality, and the ability to build social relationships, the role of such factors in influencing their empathic behavior has received little attention.

In this paper, we present a computational model of empathy which allows a virtual human to exhibit different degrees of empathy. Our model is shaped by psychological models of empathy and is based on three processing steps that are central to empathy [4]: First, the Empathy Mechanism by which an empathic emotion is produced. Second, the Empathy Modulation by which the empathic emotion is modulated. Third, the 
Expression of Empathy by which the virtual human's multimodal behavior is triggered through the modulated empathic emotion. The presented empathy model is applied and evaluated in the context of a conversational agent scenario involving the virtual humans MAX [12] and EMMA [6] and a human interaction partner. Within this scenario, our model is realized for EMMA and allows her to empathize with MAX's emotions during his interaction with the human partner.

The paper is structured as follows: In Section 2, we outline related work on existing empathic artificial agents. In Section 3, we present our approach to model empathy for a virtual human. In Section 4, we present the application scenario of the model as well as the results of an empirical evaluation of the empathic behavior generated by the model. Finally, in Section 5, we summarize the key contribution of our research.

\section{Related Work}

In previous research, much effort has gone in endowing virtual humans with the ability to empathize. McQuiggan et al. [13] propose an inductive framework for modeling parallel and reactive empathy. They called their framework CARE (Companion Assisted Reactive Empathizer) and based it on learning empirically informed models of empathy during human-agent social interactions. In a learning phase, users' situation data, such as their actions and intentions, users' affective states, bio-potential signals, and other characteristics such as their age and gender are gathered while they interact with virtual characters. The virtual characters respond to the user's situation with either parallel or reactive empathy. During interaction with the characters, users are able to evaluate their empathic responses using a 4 point Likert scale. Naive Bayes classifiers, decision trees, and support vector machines are used to learn models of empathy from 'good examples'. The induced models of empathy are used at runtime in a test phase to drive virtual characters' empathic responses. The evaluation of the characters' empathic behavior according to collected training and test data shows that the induced empathy models produce appropriate empathic behaviors.

Based on an empirical and theoretical approach, Ochs et al. [14] propose a computational model of empathic emotions. They analyzed human-machine dialog situations to identify the characteristics of dialog situations that may elicit users' emotions during human-machine interaction. The results of this empirical analysis were combined with a theoretical model of emotions to provide a model of empathic emotions. Once the user's potential emotion is determined, the agent's empathic emotion from the same type is triggered toward the user. They define a degree of empathy as a value that affects the base intensity of the empathic emotion depending on both the liking relationship between the user and the agent and the degree to which a user deserves or doesn't deserve his immediate situation (cf. [15]). The empathic behavior of the agent is empirically evaluated based on three conditions, a non-emotional condition, an empathic condition, and a non-congruent emotional condition where the agent expresses emotions that are opposite in their values of valence to the empathic emotions. The results show that the agent is perceived more positively in the empathic version and more negatively in the non-congruent emotional version. 
Rodrigues et al. [18] propose a generic computational model of empathy. Their model is integrated into an existing affective agent architecture [9] and comprises an empathic appraisal component and an empathic response component. A perceived event by an agent that evokes an emotional cue in another agent is input to the empathic appraisal component together with the emotional cue. The emotional cue is input to an emotion recognition module and the event is input to a self-projection appraisal module. The outputs of both modules are combined to determine an empathic emotion as the output of the empathic appraisal. The empathic emotion is modulated by several factors (cf. [8]). Similarity is defined as the degree of congruence of the emotions provided by the self-projection appraisal and emotion recognition modules. Affective link is defined as the value of liking between the agents. The higher the average value of similarity and affective link, the higher the value of intensity of the empathic emotion. Mood is defined as the empathizing agent's mood which then affects the intensity of the empathic emotion as it affects that of other emotions (cf. [9]). Personality refers to the empathizing agent's resistance to feel particular emotions. Regarding the empathic response component, the empathic emotion generated by the empathic appraisal triggers a situation-appropriate action. The authors designed a small scenario with four synthetic characters to evaluate their model based on two conditions, an empathy condition and a no-empathy condition. The results show that the perceived values of empathy and affective link are significantly higher in the empathy condition and are thus in line with the theoretical assumptions underlying the model.

While significant advances have been made in modeling empathy for virtual humans, the modulation of the empathic emotion and the calculation of a degree of empathy have received little attention. Accordingly, we consider the modulation of an empathic emotion and the calculation of different degrees of empathy as a crucial aspect in further enhancing an artificial agent's social behavior. While in [18] and [14] only the intensity of an empathic emotion is modulated, we also modulate its related emotion category in our model. In this regard, we follow Hoffman's claim [11] that an empathic response need not be a close match to the affect experienced by the other, but can be any emotional reaction compatible with the other's situation. Furthermore, in previous research, evaluations have been based on either two conditions, non-empathic vs. empathic (e.g., [18]) or on three conditions, non-empathic/emotional, empathic, and noncongruent empathic/emotional (e.g., [14] and [1]). In contrast, we evaluated our model based on three different conditions that distinguished three different degrees of empathy, neutral, medium, and maximum empathy, thus allowing for a more fine-grained evaluation of the model and its underlying parameters.

\section{A Computational Model of Empathy}

The virtual humans MAX [12] and EMMA [6] have a cognitive architecture composed of an emotion simulation module [2] and a Belief-Desire-Intention (BDI) module [12]. The emotion simulation module comprises of a dynamics/mood component for the calculation of the course of emotions and moods over time and their mutual interaction, and of a Pleasure, Arousal, Dominance (PAD) space in which emotion categories are located and their intensity values can be calculated. The emotion simulation module 
outputs values of pleasure, arousal, and one of two possible values of dominance (dominant vs. submissive) as well as intensity values of emotion categories. Our computational model of empathy is integrated within the emotion simulation module. In the following, we briefly introduce the three processing steps underlying our model (cf. Section 1); (more details on parts of the model and on its theoretical foundation are available in previous work [4]).

\subsection{Empathy Mechanism}

In line with the Facial Action Coding System (FACS) [10], 44 Action Units (AUs) have been implemented for the virtual human EMMA's face. In an empirical study, a total of 3517 randomly generated facial expressions of EMMA were rated by 353 participants with Pleasure, Arousal, and Dominance (PAD) values. Based on these ratings, three dimensional regression planes of AUs' intensity values and PAD values were obtained and show the meaning of each AU in PAD space. By combining all planes of all AUs, a repertoire of facial expressions arranged in PAD space was reconstructed. Accordingly, based on her own AUs and their intensity functions (regression planes) in PAD space, EMMA maps a perceived facial expression to AUs with corresponding intensity values and subsequently infers its related emotional state as a PAD value. The inferred PAD value is represented by an additional reference point in EMMA's PAD emotion space. Its related emotion category and corresponding value of intensity can thus be inferred.

After detecting a fast and salient change in the other's emotional state which indicates the occurrence of an emotional event, an empathic emotion is elicited. That is, with respect to a predetermined short time interval $T$, the difference between inferred PAD values corresponding to the time-stamps $t_{k-1}$ and $t_{k}$, with $t_{k}-t_{k-1}<=T$, is calculated as $\left|P A D_{t_{k}}-P A D_{t_{k-1}}\right|$. If this exceeds a saliency threshold $T H 1$ or if $\left|P A D_{t_{k}}\right|$ exceeds a saliency threshold $T H 2$, then the emotional state $P A D_{t_{k}}$ and its related emotion category represent the empathic emotion. The threshold values can be interpreted as representing the virtual human's responsiveness to the other's situation (for more details on the empirical study and the Empathy Mechanism see [6] and [4]). Once an empathic emotion is elicited, the following processing step Empathy Modulation is triggered.

\subsection{Empathy Modulation}

The modulation of the empathic emotion is realized within PAD space of the virtual human's emotion simulation module. At each point in time an empathic emotion is elicited, the following equation is applied:

$$
\begin{array}{r}
\text { empEmo }_{t, \text { mod }}=o w n E m o_{t}+ \\
\left(\text { empEmo }_{t}-\text { ownEmo }_{t}\right) \cdot\left(\sum_{i=1}^{n} p_{i, t} \cdot w_{i}\right) /\left(\sum_{i=1}^{n} w_{i}\right)
\end{array}
$$

The value empEmo ${ }_{t, \text { mod }}$ represents the modulated empathic emotion. The value ownEmo $_{t}$ represents the virtual human's own emotional state as the modulation factor mood. The value empEmo $t$ represents the non-modulated empathic emotion resulting from Empathy Mechanism. The values $p_{i, t}$ represent modulation factors that can 
have values ranging in $[0,1]$. The values $w_{i}$ represent assigned values of weights for the modulation factors $p_{i, t}$ which also range in $[0,1]$. Such modulation factors are, e.g, liking and familiarity which can be represented by values ranging in $[0,1]$ from neither like nor dislike to maximum like and from non-familiar to most-familiar (cf. [15]). Note that, currently, negative values of $p_{i, t}$ are not considered in our model.

We define the degree of empathy as the degree of similarity between the modulated empathic emotion and the non-modulated one. Thus, the degree of empathy is represented by the distance between empEmot,mod and empEmo $t$ within PAD space (Fig. 1, left). That is, the closer empEmo The less close empEmo

Following [18], the more similar the virtual human's emotional state to the empathic emotion, the more sensitive the virtual human to the empathic emotion. The less similar its emotional state to the empathic emotion, the more resistant the virtual human to the empathic emotion. That is, the closer the virtual human's own emotional state ownEmo to the empathic emotion empEmo the higher the resulting degree of empathy. The less close the virtual human's own emotional state ownEmo $_{t}$ to the empathic emotion

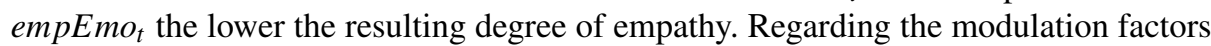
$p_{i, t}$, the higher their value of weighted mean, the closer the modulated empathic emotion empEmo t,mod $_{\text {, }}$ to the non-modulated empathic emotion empEmo ${ }_{t}$ and the higher the degree of empathy. The lower their value of weighted mean, the less close the modulated empathic emotion empEmo $o_{t, m o d}$ to the non-modulated empathic emotion empEmo $t$ and the lower the degree of empathy.

According to Hoffman [11], an empathic response to the other's emotion should be more appropriate to the other's situation than to one's own and need not be a close match to the affect experienced by the other, but can be any emotional reaction compatible with the other's situation. Further, according to the thesis of the dimensional theory [19], emotions are related to one another in a systematic manner and their relationships can be represented in a dimensional model. Accordingly, the modulated empathic

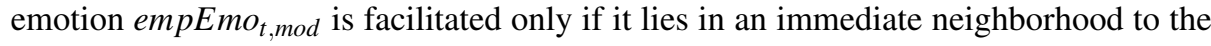
non-modulated empathic emotion $\mathrm{empEmo}_{t}$. Hence, for each emotion category located within PAD space of the emotion simulation module, we defined a so called empathy facilitation region as a box surrounding the emotion category. For example, Fig. 3 shows the PA space of positive dominance of the emotion simulation module with the defined empathy facilitation region for the emotion category annoyed. As depicted in Fig. 3 (middle), the modulated empathic emotion empEmo t,mod $_{\text {, }}$ has as related emotion category concentrated (neutral emotional state) and the non-modulated empathic emotion empEmo $t$ has as related emotion category annoyed. Accordingly, once the modulated empathic emotion empEmo emod $_{t}$ enters the empathy facilitation region defined for annoyed, it is facilitated or otherwise it is inhibited (e.g., Fig. 3, left). Within the empathy facilitation region, the modulated empathic emotion empEmo emod $_{\text {, }}$ represents an empathic response that is compatible with the other's situation (cf. [11]). Thus, the virtual human is allowed to react with an emotion from a different emotion category (but compatible) with the other's emotion.

As mentioned earlier in this section, the degree of empathy is represented by the dis-

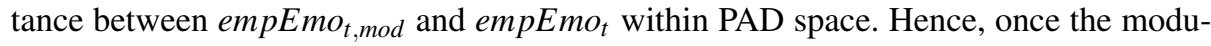




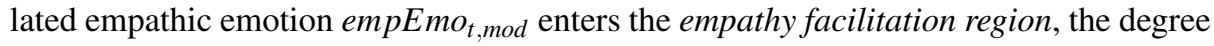
of empathy is calculated and increases toward the non-modulated empathic emotion

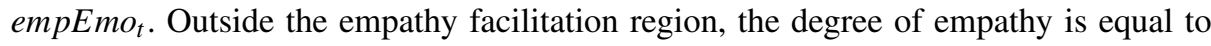
0 (Fig. 1, right). Within the empathy facilitation region, the degree of empathy is calculated by the following equation for each instance at time $t$ a modulated empathic emotion empEmot,mod is facilitated:

$$
\operatorname{degEmp}_{t}=\left(1-\left\|\frac{\text { empEmo }_{t, \text { mod }}-\text { empEmo }}{\operatorname{maxDistBox}}\right\|\right)^{2}
$$

The value $\operatorname{degEmp} p_{t}$ represents the calculated degree of empathy and ranges within $[0,1]$. The value maxDistBox represents the possible maximum distance between the

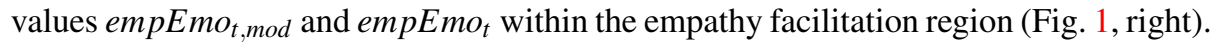
Note that the distances $\|$ empEmo t,mod $_{\text {- empEmo }} \|$ and maxDistBox are weighted distances in PAD space. That is, we defined values of weights for each dimension within PAD space. A polynomial function is chosen in order to get smooth values of the calculated degree of empathy. According to the dimensional theory [19], the pleasure dimension is the most agreed upon dimension, the arousal dimension is the second agreed upon dimension and the dominance dimension is the third and least agreed upon dimension. Thus, regarding the defined values of weight for each dimension within PAD space, we assigned a higher weight value to the pleasure dimension, a lower value to the arousal dimension, and a very low value to the dominance dimension.
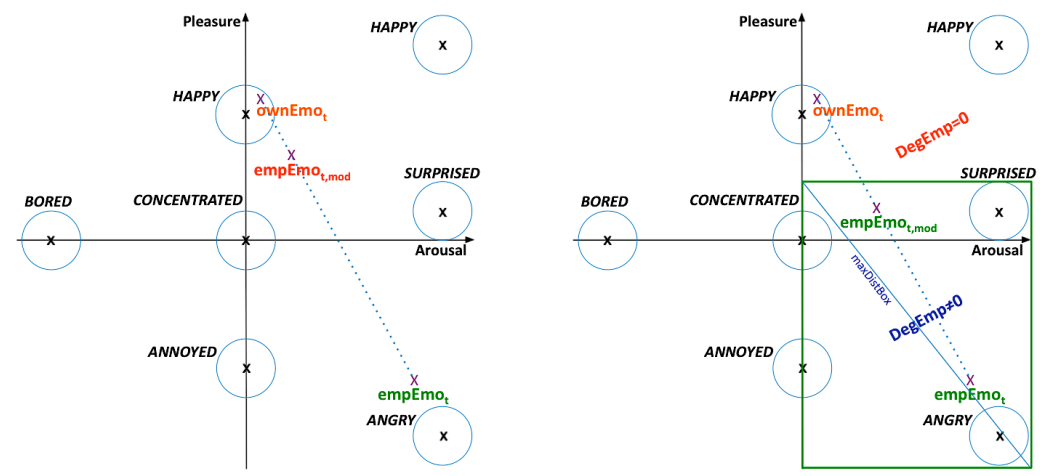

Fig. 1: The PA space of positive dominance of the emotion simulation module [2]. Left: empEmo ${ }_{t, \text { mod }}$ as lying on the straight line spanned by $o w n E m o_{t}$ and $e m p E m o_{t}$ (cf. (1)). Right: The empathy facilitation region defined for angry and the degree of empathy within PAD space.

As long as no further empathic emotion is elicited, the modulated empathic emotion represented within the virtual human's emotion module decays over time (cf. [2]). The decay function of the modulated empathic emotion is influenced by the degree of empathy, that is, the higher the calculated value of the degree of empathy, the slower the decay. The lower the value the faster the modulated empathic emotion decays. Once 
the modulated empathic emotion is facilitated, the next processing step Expression of Empathy is triggered.

\subsection{Expression of Empathy}

The modulated empathic emotion triggers EMMA's multimodal behavior as her expression of empathy. That is, EMMA's facial expression [6] and speech prosody [20] are modulated by the PAD value of her empathic emotion. The triggering of other modalities such as verbal utterances depends on the scenario's context.

\section{Application and Evaluation}

The empathy model is applied and evaluated in a conversational agent scenario where the virtual humans MAX and EMMA can engage in a multimodal small talk dialog with a human partner using speech, gestures, and facial behaviors [4] (Fig. 2, a). In this scenario, the emotions of both agents can be triggered positively or negatively by the human partner through, e.g., compliments or politically incorrect verbal utterances. During interaction, EMMA directs her attention to the speaking agent. When attending to MAX, EMMA's empathy process is triggered in response to MAX's facial expression of emotion. At each point in time, EMMA maps perceived values of MAX's facial muscles to her AUs and infers their related PAD value as MAX's perceived emotional state. Once an empathic emotion is elicited (cf. Section 3.1), it is modulated by EMMA's mood and her predefined values of liking and familiarity with MAX thus resulting in different degrees of empathy of EMMA with MAX (cf. Section 3.2). To investigate how the empathic behavior produced by our model is perceived by human participants, we conducted an empirical evaluation [5] of the model to test the following hypotheses, H1: EMMA's expression of empathy is perceivable by the participants, H2: EMMA's expressed degree of empathy is perceivable by the participants, H3: the human participants acknowledge different values of relationship between EMMA and MAX according to EMMA's expressed degree of empathy.

\subsection{Design and Procedure}

We designed 24 dialog interactions between EMMA, MAX, and a human partner (Lisa) (Fig. 2, b). At the beginning of each dialog interaction, the virtual humans are in a neutral emotional state. In each dialog interaction, Lisa begins by greeting EMMA and then praising her. Consequently, EMMA's positive emotional state happy is triggered. Simultaneously, EMMA greets Lisa and thanks her for being kind. Then Lisa greets MAX but proceeds to insult him. Thus, MAX's negative emotional state angry is triggered. Simultaneously, MAX responds with a negative verbal utterance such as "Lisa, you are horrible!". Meanwhile, EMMA empathizes with MAX to different degrees depending on her mood and her defined relationship to MAX. Note that MAX's facial expression of anger is interpreted by EMMA as showing the emotional state annoyed (cf. Section 3.1). Accordingly, the elicited empathic emotion empEmo thas as related emotion category annoyed (Fig. 3). 


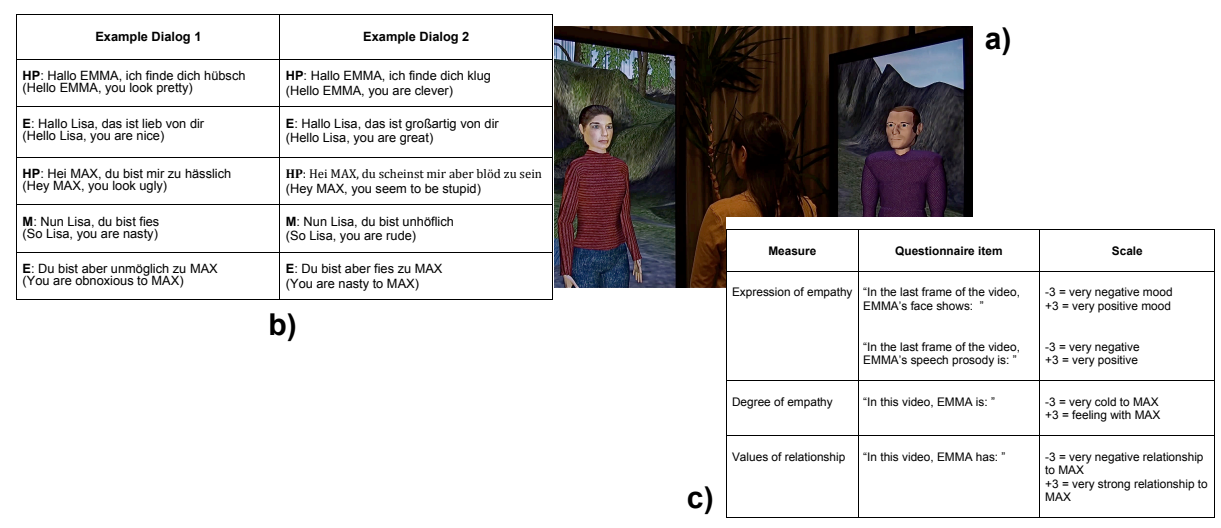

Fig. 2: a) MAX and EMMA displayed on two panels in the conversational agent scenario. b) Two example dialogs between Human Partner (HP), EMMA (E), and MAX (M). c) Schematic overview of the questionnaire. We used a 7-point Likert scale ranging from -3 to +3 .

Each dialog interaction appeared in three conditions. To create the conditions we manipulated (within-subjects) the value of EMMA's and MAX's relationship, and accordingly EMMA's degree of empathy with MAX. EMMA was in the same positive mood because she was always first complimented by Lisa (this kept the modulation factor mood constant in all three conditions). We created the three conditions by manipulating the factor liking:

1. In a first condition (neutral liking condition, Fig. 3, left), EMMA's value of liking toward MAX is set to 0. This inhibits EMMA's modulated empathic emotion and her degree of empathy equals 0 . Thus, EMMA continues in the positive emotional state happy triggered by Lisa's praise.

2. In a second condition (medium liking condition, Fig. 3, middle), EMMA's value of liking toward MAX is set to 0.5 . This facilitates her modulated empathic emotion which has as its related emotion category concentrated. EMMA's degree of empathy equals 0.25 , and she expresses the modulated empathic emotion. EMMA's values of degree of empathy and liking are higher than in the first condition.

3. In a third condition (maximum liking condition, Fig. 3, right), EMMA's value of liking toward MAX is set to 1 . As a result, her modulated empathic emotion equals the non-modulated one (with the related emotion category annoyed). EMMA in this case expresses the non-modulated empathic emotion and her value of degree of empathy equals 1 . EMMA's values of liking and degree of empathy are higher than in the other two conditions.

EMMA's facial expression and speech prosody expressed her degree of empathy. By contrast, the verbal utterance was identical in the three conditions. After MAX's response to Lisa, EMMA responded always with a negative verbal utterance (e.g., "You 


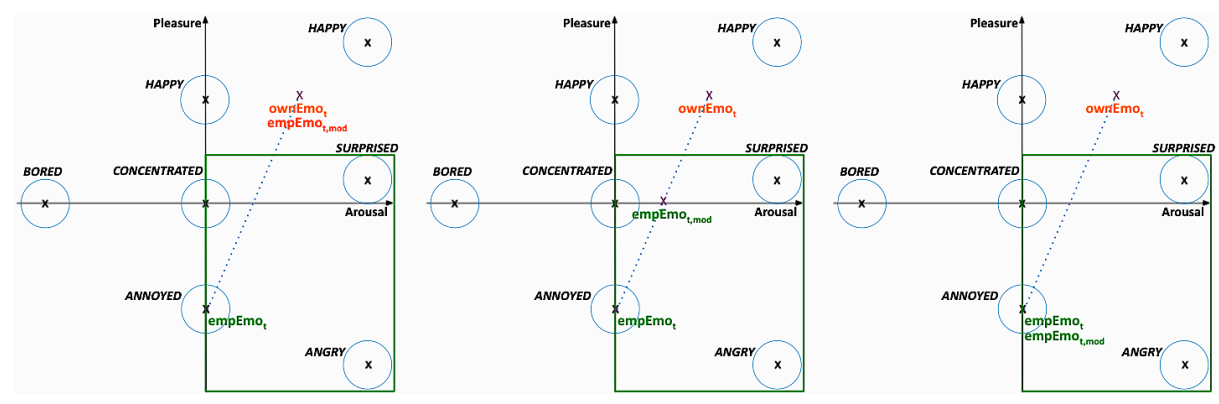

Fig. 3: The empathy facilitation region defined for annoyed represented as a box surrounding the emotion category. Left: neutral, middle: medium, right: maximum liking condition.

are nasty to MAX!", Fig. 2, b). Other behaviors of the virtual humans such as breathing, eye blinking, and conversational gestures were deactivated in all three conditions.

A total of 72 videos of the 24 dialog interactions in the three conditions were recorded. We constructed three experimental lists following a Latin Square design such that each dialog appeared in each list in only one condition. A total of 30 participants took part in the experiment, with each list assigned to 10 participants. The 24 videos contained in a list were presented in a random order to each corresponding participant. To test our three hypotheses, each participant was asked to complete a questionnaire after each presented video (Fig. 2, c).

\subsection{Results}

For the analyses of the data, we calculated the mean rating by condition for each of the four questionnaire items for participants and items (i.e. videos) separately. Next, we performed omnibus repeated measures one-way ANOVAs using participants and items as random effects. The results of the omnibus ANOVAs show a significant effect of condition for all four questionnaire items. To assess how the conditions differ from each other, we next performed a series of planned pairwise comparisons.

Expression of Empathy The mean values show that EMMA's facial expression was rated as showing a positive mood in the neutral liking condition $(M=0.883)$, as showing a slightly negative mood in the medium liking condition $(M=-0.438)$, and as showing a more negative mood in the maximum liking condition $(M=-1.554)$ (Fig. 4). Regarding her speech prosody, the mean values show that it was rated as slightly positive in the neutral liking condition $(M=0.521)$, as slightly negative in the medium liking condition $(M=-0.550)$, and as more negative in the maximum liking condition $(M=-1.592)$ (Fig. 4). The pairwise comparisons show that the three conditions were rated as significantly different from each other for facial expression $(p<.001)$ and speech prosody $(p<.001)$. 
Degree of Empathy The mean values show that EMMA was rated as slightly feeling with MAX in the neutral liking condition $(M=0.458)$ and as progressively more feeling with MAX in the medium liking condition $(M=0.992)$ and the maximum liking condition $(M=1.608)$ respectively (Fig. 4$)$. The pairwise comparisons show that the three conditions were rated as significantly different from each other $(p<.001)$.

Values of Relationship The mean values show that EMMA's value of relationship to MAX was rated as slightly positive in the neutral liking condition $(M=0.325)$, and as progressively more positive in the medium liking condition $(M=0.888)$ and the maximum liking condition $(M=1.442)$ respectively (Fig. 4$)$. The pairwise comparisons show that the three conditions were rated as significantly different from each other $(p<.001)$.
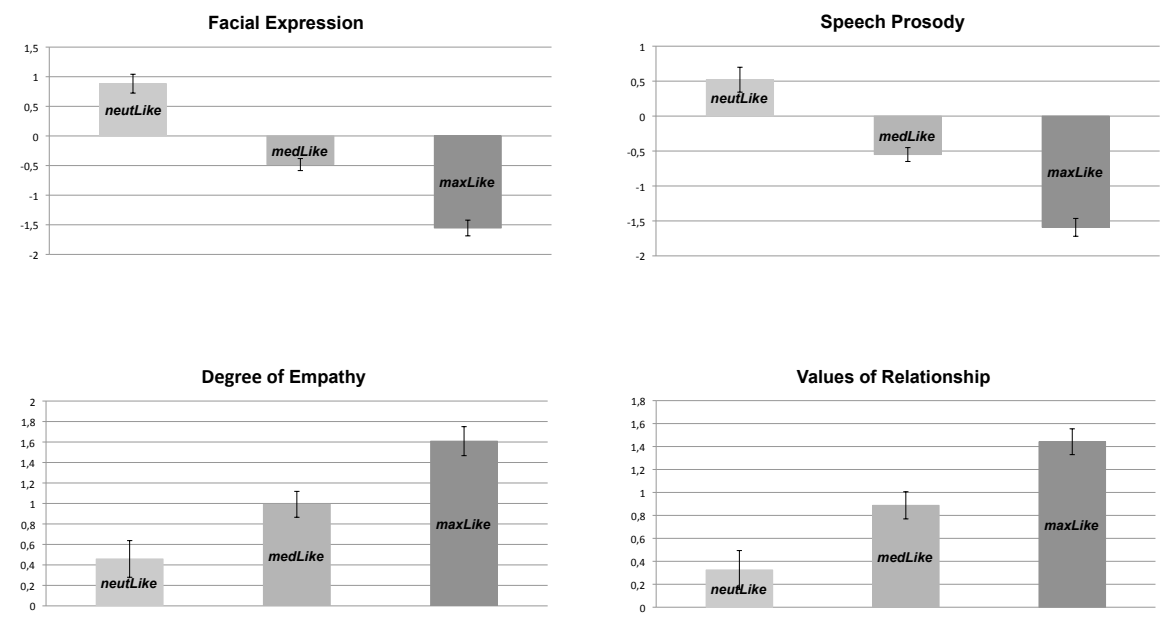

Fig. 4: Mean values and their standard errors for facial expression, speech prosody, degree of empathy, and values of relationship.

\subsection{Discussion}

The results show that EMMA's expression of empathy (facial expression and speech prosody) was appropriately recognized as positive in the neutral liking condition, and as progressively more negative in the medium and maximum liking conditions respectively. This confirms our first hypothesis H1, that EMMA's expression of empathy is perceivable by the participants, and suggests the appropriate modeling of her facial expressions and speech prosody.

The results show that EMMA's expressed degree of empathy with MAX was rated as significantly higher in the maximum liking condition than in the other two conditions, and as significantly higher in the medium liking condition than in the neutral liking condition. Hence, the results confirm our second hypothesis H2, that EMMA's 
expressed degree of empathy is perceivable by the participants. These results corroborate the theoretical assumption of our model that empathy occurs in a graded manner. The results confirmed moreover that both EMMA's facial expression and her speech prosody reliably indicate the three different empathy degrees. This corroborates our approach of modeling empathy not just as a binary function (emphatic vs. not emphatic) but rather in a graded manner that differentiates degrees of empathy.

Descriptively, Fig. 4 shows that the more EMMA's facial expression and speech prosody were rated as negative, the more EMMA was rated as empathic. That is, the more EMMA's expression of empathy was similar to MAX's expression of emotion, the more EMMA was perceived as empathic. This is in line with our definition of the degree of empathy as the degree of similarity between one's empathic emotion and the other's perceived emotion. That is, the more similar one's empathic emotion to the other's perceived emotion, the higher the degree of empathy.

Our analyses of the data showed that EMMA's different relationship values with MAX were perceived as such by the participants. The virtual humans' relationship was rated significantly higher in the maximum liking condition compared with the other two conditions, and also significantly higher in the medium than in the neutral liking condition. Overall, these results confirm hypothesis $\mathbf{H 3}$, which was that participants can perceive these subtle relationship differences that manifest through EMMA's speech prosody and facial expression. Descriptively, Fig. 4 shows that the higher they rated EMMA's expressed degree of empathy, the higher they rated EMMA's value of relationship to MAX. This is in line with our definition of the impact of relationship modulation factors in our model, e.g., liking or familiarity. That is, the higher the values of such modulation factors, the higher the similarity between the empathic emotion and the other's perceived emotion, the higher the degree of empathy. These findings further substantiate the theoretical assumption underlying our proposed model that empathy is modulated by several modulation factors such as the relationship between the empathizer and the observed other. Again, the results also show that both EMMA's facial expression and her speech prosody reliably indicate her different values of relationship to MAX thus providing further support for their appropriate modeling.

\section{Conclusion}

In this paper, we presented a computational model of empathy by which a virtual human can exhibit different degrees of empathy, an aspect that received little attention in previous research. In our model, regions of immediate neighborhood for each emotion category located in PAD space were defined. Accordingly, we defined the degree of empathy as the degree of similarity between a modulated empathic emotion and a non-modulated one within these defined regions. Hence, we exploited the assumed relationships between emotions in PAD space [19]. Note that the choice of the values of parameters in our model is a matter of design and evaluation. The findings of the empirical evaluation show that the virtual human EMMA is perceived as capable of exhibiting different degrees of empathy and values of relationship with MAX and thus warrants the conclusion that our model enhances a virtual human's social behavior. 


\section{Acknowledgments}

This research is kindly supported by the Deutsche Forschungsgemeinschaft (DFG) in the Collaborative Research Center 673. This paper is a preprint version of an article published by Springer-Verlag. The original publication is available at http://link. springer. com/chapter/10.1007/978-3-642-40942-4_3.

\section{References}

1. C. Becker, H. Prendinger, M. Ishizuka, and I. Wachsmuth. Evaluating affective feedback of the 3D agent Max in a competitive cards game. In Affective Computing and Intelligent Interaction (ACII 2005), pages 466-473, Beijing, China, 2005. Springer, Berlin.

2. C. Becker-Asano and I. Wachsmuth. Affective computing with primary and secondary emotions in a virtual human. Autonomous Agents and Multi-Agent Systems, 20(1):32-49, 2010.

3. T. Bickmore and R. Picard. Establishing and maintaining long-term human-computer relationships. ACM Transactions on Computer-Human Interaction (TOCHI), 12(2):293-327, 2005.

4. H. Boukricha and I. Wachsmuth. Empathy-based emotional alignment for a virtual human: A three-step approach. Künstl Intell, 25(3):195-204, 2011.

5. H. Boukricha, I. Wachsmuth, M. Carminati, and P. Knoeferle. A computational model of empathy: Empirical evaluation (in press). In Affective Computing and Intelligent Interaction (ACII 2013), Geneva, Switzerland, 2013.

6. H. Boukricha, I. Wachsmuth, A. Hofstätter, and K. Grammer. Pleasure-arousal-dominance driven facial expression simulation. In 3rd International Conference on Affective Computing and Intelligent Interaction (ACII), pages 119-125, Amsterdam, Netherlands, 2009. IEEE.

7. S. Brave, C. Nass, and K. Hutchinson. Computers that care: investigating the effects of orientation of emotion exhibited by an embodied computer agent. International Journal of Human-Computer Studies, 62:162-178, 2005.

8. F. de Vignemont and T. Singer. The empathic brain: how, when and why? Trends in Cognitive Sciences, 10(10):435-441, 2006.

9. J. Dias and A. Paiva. Feeling and reasoning: A computational model for emotional characters. In C. Bento, A. Cardoso, and G. Dias, editors, EPIA'05 Proceedings of the 12th Portuguese conference on Progress in Artificial Intelligence, volume LNAI 3808, pages 127140. Springer-Verlag Berlin Heidelberg, 2005.

10. P. Ekman, W. V. Friesen, and J. C. Hager. Facial Action Coding System: Investigator's Guide. Research Nexus, a subsidiary of Network Information Research Corporation, Salt Lake City UT, USA, 2002.

11. M. L. Hoffman. Empathy and Moral Development. Cambridge University Press, 2000.

12. N. Lessmann, S. Kopp, and I. Wachsmuth. Situated interaction with a virtual human - perception, action, and cognition. In G. Rickheit and I. Wachsmuth, editors, Situated Communication, pages 287-323. Mouton de Gruyter, Berlin, 2006.

13. S. McQuiggan, J. Robison, R. Phillips, and J. Lester. Modeling parallel and reactive empathy in virtual agents: An inductive approach. In L. Padgham, D. C. Parkes, J. Mueller, and S. Parsons, editors, Proc. of 7th Int. Conf. on Autonomous Agents and Multiagent Systems (AAMAS 2008), pages 167-174, Estoril, Portugal, 2008.

14. M. Ochs, D. Sadek, and C. Pelachaud. A formal model of emotions for an empathic rational dialog agent. Autonomous Agents and Multi-Agent Systems, 24(3):410-440, 2012.

15. A. Ortony, G. Clore, and A. Collins. The Cognitive Structure of Emotions. Cambridge University Press, 1988. 
16. A. Paiva, J. Dias, D. Sobral, R. Aylett, S. Woods, L. Hall, and C. Zoll. Learning by feeling: Evoking empathy with synthetic characters. Applied Artificial Intelligence, 19:235-266, 2005.

17. H. Prendinger and M. Ishizuka. The empathic companion: A character-based interface that adresses users' affective states. Applied Artificial Intelligence, 19:267-285, 2005.

18. S. H. Rodrigues, S. Mascarenhas, J. Dias, and A. Paiva. I can feel it too! : Emergent empathic reactions between synthetic characters. In 3rd International Conference on Affective Computing and Intelligent Interaction (ACII), Amsterdam, Netherland, 2009. IEEE.

19. J. Russell and A. Mehrabian. Evidence for a three-factor theory of emotions. Journal of Research in Personality, 11(3):273-294, 1977.

20. M. Schröder and J. Trouvain. The German text-to-speech system MARY: A tool for research, development and teaching. International Journal of Speech Technology, 6(4):365-377, 2003. 